\title{
On Optimal Bilinear Quadrilateral Meshes
}

\author{
Ed D'Azevedo*
}

\begin{abstract}
.
The novelty of this work is in presenting interesting error properties of two types of asymptotically "optimal" quadrilateral meshes for bilinear approximation. The first type of mesh has an error equidistributing property where the maximum interpolation error is asymptotically the same over all elements. The second type has faster than expected "super-convergence" property for certain saddleshaped data functions. The "super-convergent" mesh may be an order of magnitude more accurate than the error equidistributing mesh. Both types of mesh are generated by a coordinate transformation of a regular mesh of squares. The coordinate transformation is derived by interpreting the Hessian matrix of a data function as a metric tensor. The insights in this work may have application in mesh design near known corner or point singularities.
\end{abstract}

keywords. anisotropic mesh, quadrilateral mesh, mesh generation, super-convergence.

\section{Introduction}

This paper presents the theoretical effectiveness of two types of "optimal" bilinear quadrilateral meshes. The novelty of this work is in presenting interesting error properties of two types of asymptotically "optimal" quadrilateral meshes for bilinear approximation. The first type of mesh has an error equidistributing property where the maximum interpolation error is asymptotically the same over all elements. The second type has faster than expected "super-convergence" property for certain non-convex saddle-shaped data functions. The "super-convergent" mesh may be an order of magnitude more accurate than the error equidistributing mesh. Both types of meshes are generated by a coordinate transformation of a regular mesh of squares. The coordinate transformation is derived by interpreting the Hessian matrix of a data function as a metric tensor. This work is a basic study on optimal meshes with the intention of gaining insight into the more complex meshing problem in surface approximation and finite element analysis especially near corner or point singularities.

For simplicity, we consider the problem of interpolating a given smooth data function with continuous piecewise bilinear quadrilaterals over a domain to satisfy a given error tolerance. A mesh that achieves this error tolerance with the fewest elements is defined to be optimally efficient. Intuitively, one would expect smaller and denser elements in regions where the function has sharp peaks or large variations.

Provably optimal triangular meshes $[2,4]$ have been produced by anisotropic mesh transformation. Anisotropic mesh transformation is emerging as an effective technique for unstructured grid generation where the vertex distribution is highly non-uniform. The central idea is to control the element shapes and sizes by specifying a symmetric metric tensor that measures the approximation error. The metric tensor determines the corresponding anisotropic transformation. The anisotropic mesh is then the image of a uniform mesh of optimal shape elements under the anisotropic transformation. Simpson [9] gives a survey on anisotropic meshes. Nadler [6], D'Azevedo and Simpson [3, 4], and D'Azevedo [2] have studied local anisotropic transformation for generating optimally efficient triangular meshes. Numerous works such as Borouchaki [1], Peraire [7], and Shimada [8], have used the Hessian matrix as a metric tensor for anisotropic mesh generation. In this paper we apply a similar analysis to bilinear approximation on quadrilateral patches.

An outline of the paper follows. In $\S 2$, we present a simple local quadratic model for error analysis and introduce the coordinate transformation to the "isotropic" space. In $\S 3$ we show a square over the isotropic space is the most efficient shape to minimize the ratio of Error/Area. A regular mesh of squares over the isotropic space would correspond to an optimally efficient mesh in the original space. Section 4 states a classical result in differential geometry on the conditions for finding the anisotropic transformation $[\tilde{x}(x, y), \tilde{y}(x, y)]$ for a general data function. Results of numerical experiments are presented in $\S 5$ to demonstrate the error equidistributing property and the effectiveness of the super-convergent meshes.

*Computer Science and Mathematics Division, Oak Ridge National Laboratory, P. O. Box 2008, Oak Ridge, TN $37831-6367$. Work was funded in part by the $\Lambda$ pplied Mathematical Sciences Research Program, Office of Energy Research, U.S. Department of Energy undcr contract DE-AC05-96OR22464 with Lockheed Martin Energy Research Corp. This submitted manuscript has been authored by a contractor of the U. S. Government under Contract No. DE-AC05-96OR22464. Accordingly, the U. S. Government retains a nonexclusive, royalty-free license to publish or reproduce the published form of this contribution, or allow others to do so, for U. S. Government purposes.

Oak Ridge National Laboratory is managed by Lockheed Martin

Energy Research Corporation for the United States Department

of Energy under Contract No. DE-AC05-96OR22464. 


\section{Quadratic model}

We shall consider a local analysis where we assume the data function $f(x, y)$ in the neighborhood of $\left(x_{c}, y_{c}\right)$ is well approximated by its quadratic Taylor expansion,

$$
\begin{aligned}
f(x, y) & =f\left(x_{c}+d x, y_{c}+d y\right) \\
& \approx f\left(x_{c}, y_{c}\right)+\nabla f\left(x_{c}, y_{c}\right)[d x, d y]+\frac{1}{2}[d x, d y] H[d x, d y]^{t}
\end{aligned}
$$

The function is convex if $\operatorname{det}(H)>0$ and saddle-shaped if $\operatorname{det}(H)<0$. The key insight in [2] is in interpreting the Hessian matrix $H$ in (1) as a symmetric metric tensor. Let the symmetric Hessian matrix be diagonalizable as

$$
\begin{aligned}
& H=Q^{t}\left[\begin{array}{cc}
\lambda_{1} & 0 \\
0 & \lambda_{2}
\end{array}\right] Q=S^{t}\left[\begin{array}{cc}
1 & 0 \\
0 & \epsilon
\end{array}\right] S, \quad \text { where } \epsilon=\operatorname{sign}(\operatorname{det}(H)), \\
& S=\left[\begin{array}{cc}
\sqrt{\left|\lambda_{1}\right|} & 0 \\
0 & \sqrt{\left|\lambda_{2}\right|}
\end{array}\right] Q, \quad \text { and } Q \text { is orthogonal, } Q^{t} Q=I
\end{aligned}
$$

Note that transformation $S$ is essentially a rotation to align eigenvectors along the coordinate axes then followed by a simple scaling. Under this transformation $S$, the expression $[d x, d y] H[d x, d y]^{t}$ reduces to $(d \tilde{x})^{2}+\epsilon(d \tilde{y})^{2}$, where $[\tilde{x}, \tilde{y}]^{t}=S[x, y]^{t}$. Over the transformed space $(\tilde{x}(x, y), \tilde{y}(x, y))$, the Hessian matrix is reduced to a simple form $(2)$ with no preference for any direction. We shall call this transformed space the "isotropic" space. We shall use a quadratic data function to derive a simple model for deriving the maximum interpolation error over a bilinear quadrilateral patch.

\section{Quadrilateral patch}

The bilinear interpolant over a quadrilateral element is given by the isoparametric formulation (commonly used in finite element analysis) over the normalized $(p, q)$-space on the unit square, $0 \leq p, q \leq 1$. Basis functions are

$$
\begin{array}{ll}
\phi_{1}(p, q)=(1-p)(1-q), & \phi_{2}(p, q)=p(1-q) \\
\phi_{3}(p, q)=p q, & \phi_{4}(p, q)=(1-p) q
\end{array}
$$

that satisfy $\phi_{i}\left(x_{j}, y_{j}\right)=\delta_{i j}$, and sum to one, $1=\sum_{i=1}^{i=4} \phi_{i}(p, q)$.

Mapping from $(p, q)$ to the original $(x, y)$-space is by

$$
\begin{aligned}
& x(p, q)=x_{1} \phi_{1}(p, q)+x_{2} \phi_{2}(p, q)+x_{3} \phi_{3}(p, q)+x_{4} \phi_{4}(p, q) \\
& y(p, q)=y_{1} \phi_{1}(p, q)+y_{2} \phi_{2}(p, q)+y_{3} \phi_{3}(p, q)+y_{4} \phi_{4}(p, q)
\end{aligned}
$$

that maps vertex $(0,0)$ to $\left(x_{1}, y_{1}\right)$, vertex $(1,0)$ to $\left(x_{2}, y_{2}\right),(1,1)$ to $\left(x_{3}, y_{3}\right)$ and $(0,1)$ to $\left(x_{4}, y_{4}\right)$. The bilinear interpolant (over $(p, q)$-space) is given by

$$
p_{b}(x(p, q), y(p, q))=\sum_{i=1}^{i=4} f\left(x_{i}, y_{i}\right) \phi_{i}(p, q)
$$

The error function for quadratic interpolation over a parallelogram can be shown by direct algebraic expansion (see Appendix A) to be

$$
\begin{aligned}
E_{Q}(p, q) & =p_{b}(x(p, q), y(p, q))-f(x(p, q), y(p, q)) \\
& =\mathcal{E}_{Q}-\frac{1}{2}\left(\mu_{1}\left(p-p_{c}\right)^{2}+\mu_{2}\left(q-q_{c}\right)^{2}\right)
\end{aligned}
$$

with centroid at $\left[p_{c}, q_{c}\right]=\left[\frac{1}{2}, \frac{1}{2}\right]$,

$$
\left[u_{x}, u_{y}\right]=\left[x_{2}-x_{1}, y_{2}-y_{1}\right], \quad\left[v_{x}, v_{y}\right]=\left[x_{1}-x_{1}, y_{4}-y_{1}\right]
$$




$$
\begin{aligned}
\mathcal{E}_{Q} & =E_{Q}\left(p_{c}, q_{c}\right)=\frac{1}{8}\left(\mu_{1}+\mu_{2}\right), \\
0 & =\frac{\partial}{\partial p} E_{Q}\left(p_{c}, q_{c}\right)=\frac{\partial}{\partial q} E_{Q}\left(p_{c}, q_{c}\right), \\
\mu_{1} & =\left[u_{x}, u_{y}\right] H\left[u_{x}, u_{y}\right]^{t}, \quad \mu_{2}=\left[v_{x}, v_{y}\right] H\left[v_{x}, v_{y}\right]^{t} .
\end{aligned}
$$

For a convex function $(\operatorname{det}(H)>0), \mu_{1}$ and $\mu_{2}$ are positive, hence the maximum error is attained at the centroid $\left[p_{c}, q_{c}\right]$.

For the case of a general convex quadrilateral, the error expression is more complicated. However, we can show a square over the isotropic space is of optimal shape by minimizing the efficiency ratio (Error/Area). Since the isoparametric bilinear interpolant (5) exactly fits linear functions [5], the error attained at the centroid $\left(x_{c}, y_{c}\right)$ can be written as

$$
\begin{aligned}
E_{M} & =\frac{1}{4}\left(\sum_{i=1}^{i=4} \frac{1}{2}\left[x_{i}, y_{i}\right] H\left[x_{i}, y_{i}\right]^{l}\right)-\frac{1}{2}\left[x_{c}, y_{c}\right] H\left[x_{c}, y_{c}\right]^{t} \\
& =\frac{1}{8}\left(\sum_{i=1}^{i=4}\left(\left[x_{i}, y_{i}\right] H\left[x_{i}, y_{i}\right]^{t}-\left[x_{c}, y_{c}\right] H\left[x_{c}, y_{c}\right]^{t}\right)\right) \\
{\left[x_{c}, y_{c}\right] } & =\left[\left(x_{1}+x_{2}+x_{3}+x_{4}\right) / 4,\left(y_{1}+y_{2}+y_{3}+y_{4}\right) / 4\right] .
\end{aligned}
$$

This expression can be further simplified over the isotropic space where $H$ is the identity,

$$
\begin{aligned}
\mathcal{E}_{\mathrm{M}} & =\frac{1}{8}\left(\sum_{i=1}^{i=4}\left(\left(\tilde{x}_{i}^{2}+\tilde{y}_{i}^{2}\right)-\left(\tilde{x}_{c}^{2}+\tilde{y}_{c}^{2}\right)\right)\right) \\
& =\frac{1}{8}\left(\left(\tilde{x}_{1}^{2}+\tilde{x}_{2}^{2}+\tilde{x}_{3}^{2}+\tilde{x}_{4}^{2}\right)-4 \tilde{x}_{c}^{2}+\left(\tilde{y}_{1}^{2}+\tilde{y}_{2}^{2}+\tilde{y}_{3}^{2}+\tilde{y}_{4}^{2}\right)-4 \tilde{y}_{c}^{2}\right) \\
& =\frac{1}{8}\left(L_{1}^{2}+L_{2}^{2}+L_{3}^{2}+L_{4}^{2}\right), \quad \text { with } L_{i}^{2}=\left(\tilde{x}_{i}-\tilde{x}_{c}\right)^{2}+\left(\tilde{y}_{i}-\tilde{y}_{c}\right)^{2},
\end{aligned}
$$

where $\left[\tilde{x}_{i}, \tilde{y}_{i}\right]^{t}=S\left[x_{i}, y_{i}\right]^{t}$ and $\left[\tilde{x}_{c}, \tilde{y}_{c}\right]^{t}=S\left[x_{c}, y_{c}\right]^{t}$ are the corresponding coordinates over the isotropic space. The area of this transformed convex quadrilateral is (see Figure 1)

$$
\text { Area }=\frac{1}{2}\left(L_{1} L_{2} \sin \left(\theta_{1}\right)+L_{2} L_{3} \sin \left(\theta_{2}\right)+L_{3} L_{4} \sin \left(\theta_{3}\right)-L_{4} L_{1} \sin \left(\theta_{1}+\theta_{2}+\theta_{3}\right)\right) .
$$

Since the isotropic transformation $S$ in (2) is a rotation followed by a rescaling of coordinate axis, the area of quadrilateral over the isotropic space is scaled by $\sqrt{\left|\lambda_{1} \lambda_{2}\right|}=\sqrt{|\operatorname{det}(H)|}$ (intrinsic to $H$ ). By calculus, we can show this ratio of $\mathcal{E}_{M} /$ Area is minimized and attained by a square with $L_{1}=L_{2}=L_{3}=L_{4}$ and $\theta_{1}=\theta_{2}=\theta_{3}=\pi / 4$. Hence the most efficient shape among all general convex bilinear quadrilaterals is a square over the isotropic space with an efficiency ratio of $1 / 4$.

If $f(x, y)$ is saddle-shaped $(\operatorname{det}(H)<0)$, the error expression for a parallelogram is still

$$
E_{\mathcal{Q}}(p, q)=\frac{1}{8}\left(\mu_{1}+\mu_{2}\right)-\frac{1}{2}\left(\mu_{1}\left(p-p_{c}\right)^{2}+\mu_{2}\left(q-q_{c}\right)^{2}\right) .
$$

Under the anisotropic transformation $S$,

$$
\mu_{1}=\tilde{u}_{x}^{2}-\tilde{u}_{y}^{2}, \quad \mu_{2}=\tilde{v}_{x}^{2}-\tilde{v}_{y}^{2}, \quad\left[\begin{array}{cc}
\tilde{u}_{x} & \tilde{v}_{x} \\
\tilde{u}_{y} & \tilde{v}_{y}
\end{array}\right]=S\left[\begin{array}{cc}
u_{x} & v_{x} \\
u_{y} & v_{y}
\end{array}\right] .
$$

For a square over the isotropic space, we have

$$
\begin{gathered}
{\left[u_{x}, u_{y}\right]=[L, 0], \quad\left[v_{x}, v_{y}\right]=[0, L], \quad \mu_{1}=L^{2}, \quad \mu_{2}=-L^{2},} \\
E_{Q}(p, q)=-\frac{1}{2}\left(L^{2}\left(p-\frac{1}{2}\right)^{2}-L^{2}\left(q-\frac{1}{2}\right)^{2}\right)=\frac{L^{2}}{2}\left(\left(q-\frac{1}{2}\right)^{2}-\left(p-\frac{1}{2}\right)^{2}\right) .
\end{gathered}
$$

The maximum error is $L^{2} / 8$ and attained at $(p, q)=\left(\frac{1}{2}, 1\right)$ or $\left(\frac{1}{2}, 0\right)$. 


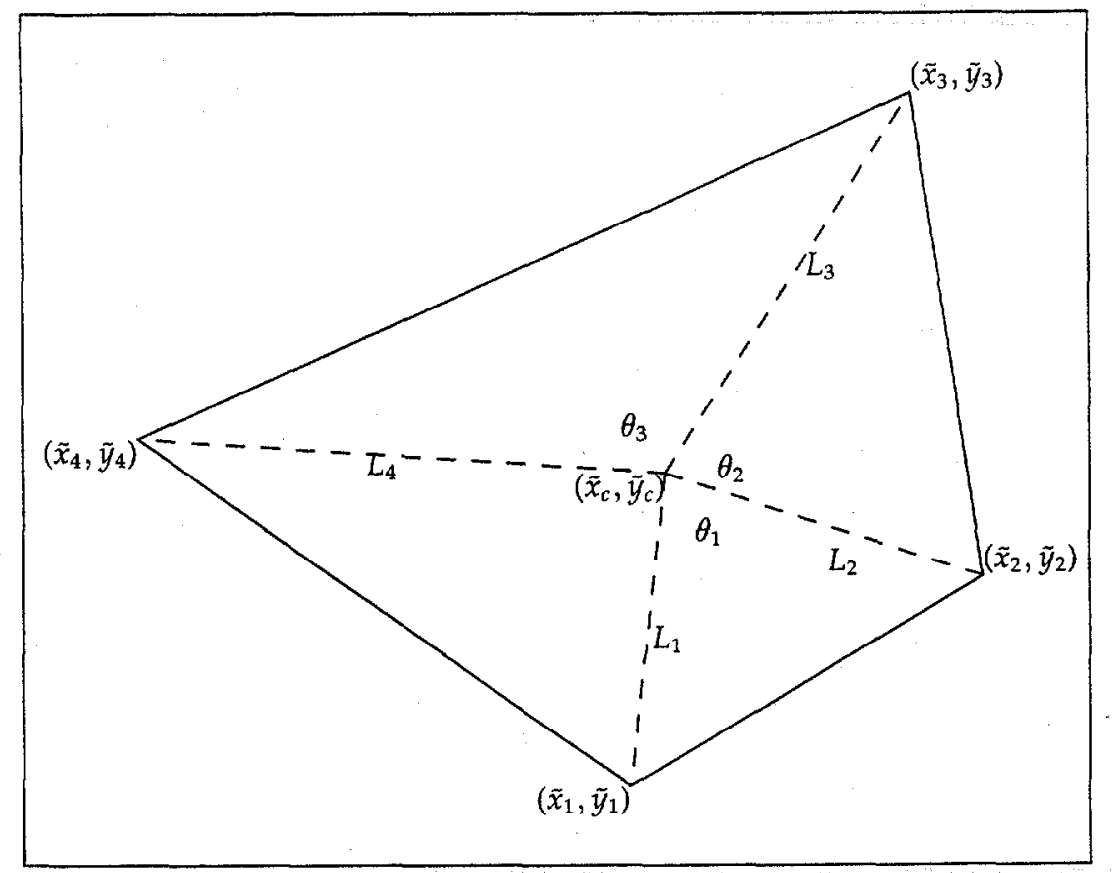

Figure 1: Convex quadrilateral over isotropic space.

Note that both $\mu_{1}$ and $\mu_{2}$ vanish for

$$
\left[\tilde{u}_{x}, \tilde{u}_{y}\right]=[L, L], \quad\left[\tilde{v}_{x}, \tilde{v}_{y}\right]=[-L, L]
$$

which correspond to a square rotated by $\pi / 4$. The above indicates an "exact fit" $\left(E_{Q}(p, q)=0\right)$ if $\mu_{1}=\mu_{2}=0$. This suggests bilinear approximation has higher than expected accuracy and the simple quadratic model is inadequate to fully capture the error properties in this case.

To summarize, a square over the isotropic space in any orientation is of optimal shape for the convex $(\operatorname{det}(H)>0)$ case, and a square rotated by $\pi / 4$ is the optimal shape for the saddle-shaped $(\operatorname{det}(H)<0)$ case. A regular square mesh over the isotropic space would correspond to a error equidistributing mesh where each patch incurs the same maximum error. For a saddle-shaped data function $\operatorname{det}(H)<0$, a regular mesh of squares rotated $\pi / 4$ would have higher than expected accuracy.

\section{Differential Geometry}

The constant Hessian Matrix $H=\left\{h_{i j}\right\}$ in (1) determines the coordinate transformation $S$ that maps $[\bar{x}, \tilde{y}]^{t}=S[x, y]^{t}$ so that

$$
[d x, d y] H[d x, d y]^{t}=d \tilde{x}^{2}+\epsilon d \tilde{y}^{2}
$$

For more general functions, we may view the Hessian matrix $H(x, y)$ as a metric tensor for measuring the interpolation error $[d x, d y] H[d x, d y]^{t}$. Thus we need to determine $[\tilde{x}(x, y), \tilde{y}(x, y)]$, a continuous transformation that globally satisfies $[d x, d y] H[d x, d y]^{t}=d \tilde{x}^{2}+\epsilon d \tilde{y}^{2}$ for infinitestimals $[d x, d y]$. The transformation $[\tilde{x}(x, y), \tilde{y}(x, y)]$ should satisfy

$$
h_{11} d x^{2}+2 h_{12} d x d y+h_{22} d y^{2}=\left(\frac{\partial \tilde{x}}{\partial x} d x+\frac{\partial \tilde{x}}{\partial y} d y\right)^{2}+\epsilon\left(\frac{\partial \tilde{y}}{\partial x} d x+\frac{\partial \tilde{y}}{\partial y} d y\right)^{2}
$$




$$
\begin{aligned}
& h_{11}=\frac{\partial^{2}}{\partial x^{2}} f(x, y)=\left(\frac{\partial \tilde{x}}{\partial x}\right)^{2}+\epsilon\left(\frac{\partial \tilde{y}}{\partial x}\right)^{2}, \\
& h_{12}=\frac{\partial^{2}}{\partial x \partial y} f(x, y)=\frac{\partial \tilde{x}}{\partial x} \frac{\partial \tilde{y}}{\partial y}+\epsilon \frac{\partial \tilde{y}}{\partial x} \frac{\partial \tilde{x}}{\partial y}, \\
& h_{22}=\frac{\partial^{2}}{\partial y^{2}} f(x, y)=\left(\frac{\partial \tilde{x}}{\partial y}\right)^{2}+\epsilon\left(\frac{\partial \tilde{y}}{\partial y}\right)^{2} .
\end{aligned}
$$

The conditions for finding the anisotropic coordinate transformation $[\tilde{x}(x, y), \tilde{y}(x, y)]$ are given by a classical result in differential geometry for characterizing a "flat" space [10]: that the Riemann-Christoffel tensor formed from the metric tensor $H$ is identically zero. In this case, a sufficient condition is for $H=\left\{h_{i j}\right\}$ to satisfy

$$
K_{1} h_{11}+K_{2} h_{12}+K_{3} h_{22}=0
$$

for some constants $K_{1}, K_{2}, K_{3}$. In particular, (13) is satisfied by harmonic functions $\left(h_{11}+h_{22}=0\right)$. The coordinate transformation $[\tilde{x}(x, y), \tilde{y}(x, y)]$ may be found by solving an initial value ordinary differential equation. The details for computing the anisotropic coordinate transformation $[\tilde{x}(x, y), \tilde{y}(x, y)]$ are described in [2].

\section{Numerical Experiments}

In this section, we demonstrate the effectiveness of a super-convergent mesh for interpolation over bilinear quadrilaterals on several harmonic functions. To clearly illustrate the error equidistributing properties, only elements entirely interior to the unit square are generated to simplify the presentation.

Example 1. A logarithmic singularity at $\left(x_{0}, y_{0}\right)=(0.5,-0.2)$,

$$
f(x, y)=\ln \left(\left(x-x_{0}\right)^{2}+\left(y-y_{0}\right)^{2}\right) / 2, \quad \operatorname{det}(H)=-\left(\left(x-x_{0}\right)^{2}+\left(y-y_{0}\right)^{2}\right)^{-2} .
$$

Coordinate transformation is

$$
\tilde{x}(x, y)=\arctan \left(y-y_{0}, x-x_{0}\right), \quad \tilde{y}(x, y)=\ln \left(\left(x-x_{0}\right)^{2}+\left(y-y_{0}\right)^{2}\right) / 2 .
$$

Example 2. A near singularity at $\left(x_{0}, y_{0}\right)=(0.5,-0.2)$,

$$
f(x, y)=\frac{\left(x-x_{0}\right)^{2}-\left(y-y_{0}\right)^{2}}{\left(\left(x-x_{0}\right)^{2}+\left(y-y_{0}\right)^{2}\right)^{2}}, \quad \operatorname{det}(H)=-36\left(\left(x-x_{0}\right)^{2}+\left(y-y_{0}\right)^{2}\right)^{-4} .
$$

Coordinate transformation is

$$
\tilde{x}(x, y)=\sqrt{6}\left(1-\frac{x-x_{0}}{\left(x-x_{0}\right)^{2}+\left(y-y_{0}\right)^{2}}\right), \quad \tilde{y}(x, y)=\sqrt{6} \frac{y-y_{0}}{\left(x-x_{0}\right)^{2}+\left(y-y_{0}\right)^{2}} .
$$

Example 3. A more severe near singularity at $\left(x_{0}, y_{0}\right)=(0.5,-0.2)$,

$$
f(x, y)=\frac{\left(\left(x-x_{0}\right)^{2}+\left(y-y_{0}\right)^{2}\right)^{2}-8\left(x-x_{0}\right)^{2}\left(y-y_{0}\right)^{2}}{\left(\left(x-x_{0}\right)^{2}+\left(y-y_{0}\right)^{2}\right)^{4}}, \quad \operatorname{det}(H)=-400\left(\left(x-x_{0}\right)^{2}+\left(y-y_{0}\right)^{2}\right)^{-6} .
$$

Coordinate transformation is

$$
\tilde{x}(x, y)=\sqrt{5}\left(1+\frac{\left(y-y_{0}\right)^{2}-\left(x-x_{0}\right)^{2}}{\left(\left(x-x_{0}\right)^{2}+\left(y-y_{0}\right)^{2}\right)^{2}}\right), \quad \tilde{y}(x, y)=2 \sqrt{5} \frac{\left(x-x_{0}\right)\left(y-y_{0}\right)}{\left(\left(x-x_{0}\right)^{2}+\left(y-y_{0}\right)^{2}\right)^{2}}
$$

Example 4. Potential flow around a corner at $\left(x_{0}, y_{0}\right)=(0.5,0.5)$ where $n=\pi / \alpha=16 / 31, \alpha=2 \pi-\pi / 16$ is the angle of corner, and $\theta=\arctan (y, x)$,

$$
f(x, y)=\left(\left(x-x_{0}\right)^{2}+\left(y-y_{0}\right)^{2}\right)^{n / 2} \cos (n \theta), \quad \operatorname{det}(H)=-\frac{57600}{923521}\left(\left(x-x_{0}\right)^{2}+\left(y-y_{0}\right)^{2}\right)^{-46 / 31} .
$$


Table 1: Summary of results for Example 1.

\begin{tabular}{|l||c|c|c|c|c|}
\hline \hline & $\begin{array}{c}\text { Minimum } \\
\text { crror }\end{array}$ & $\begin{array}{c}\text { Median } \\
\text { error }\end{array}$ & $\begin{array}{c}90 \\
\text { percentile }\end{array}$ & $\begin{array}{c}\text { Maximum } \\
\text { error }\end{array}$ & $\begin{array}{c}\text { Number of } \\
\text { elements }\end{array}$ \\
\hline \hline Mesh I & $3.56 \mathrm{e}-04$ & $3.56 \mathrm{e}-04$ & $3.56 \mathrm{e}-04$ & $3.56 \mathrm{e}-04$ & 918 \\
Mesh I & $8.90 \mathrm{e}-05$ & $8.90 \mathrm{e}-05$ & $8.90 \mathrm{e}-05$ & $8.90 \mathrm{e}-05$ & 3841 \\
Mesh I & $2.22 \mathrm{e}-05$ & $2.22 \mathrm{e}-05$ & $2.22 \mathrm{e}-05$ & $2.22 \mathrm{e}-05$ & 15674 \\
\hline Mesh II & $3.44 \mathrm{e}-06$ & $3.44 \mathrm{e}-06$ & $3.44 \mathrm{e}-06$ & $3.44 \mathrm{e}-06$ & 923 \\
Mesh II & $4.30 \mathrm{e}-07$ & $4.30 \mathrm{e}-07$ & $4.30 \mathrm{e}-07$ & $4.30 \mathrm{e}-07$ & 3847 \\
Mesh II & $5.37 \mathrm{e}-08$ & $5.37 \mathrm{e}-08$ & $5.37 \mathrm{e}-08$ & $5.37 \mathrm{e}-08$ & 15695 \\
\hline
\end{tabular}

Table 2: Summary of results for Example 2.

\begin{tabular}{|l||c|c|c|c|c|}
\hline & $\begin{array}{c}\text { Minimum } \\
\text { error }\end{array}$ & $\begin{array}{c}\text { Median } \\
\text { error }\end{array}$ & $\begin{array}{c}90 \\
\text { percentile }\end{array}$ & $\begin{array}{c}\text { Maximum } \\
\text { error }\end{array}$ & $\begin{array}{c}\text { Number of } \\
\text { elements }\end{array}$ \\
\hline \hline Mesh I & $1.30 \mathrm{e}-02$ & $1.30 \mathrm{e}-02$ & $1.30 \mathrm{e}-02$ & $1.30 \mathrm{e}-02$ & 920 \\
Mesh II & $1.27 \mathrm{e}-04$ & $1.79 \mathrm{e}-04$ & $3.18 \mathrm{e}-04$ & $6.93 \mathrm{e}-04$ & 921 \\
\hline
\end{tabular}

Coordinate transformation is

$$
[\tilde{x}(x, y), \tilde{y}(x, y)]=\frac{\sqrt{15}}{2}\left(\left(x-x_{0}\right)^{2}+\left(y-y_{0}\right)^{2}\right)^{4 / 31}[\sin (8 \theta / 31), \cos (8 \theta / 31)] .
$$

The results of the experiments are summarized in Figures 2, 3, 4,5 and in Tables 1, 2,3 and 4. Mesh I is generated by a regular mesh of squares over the isotropic space. Mesh II is generated by a regular mesh of squares but with the $\pi / 4$ rotation over the isotropic space to capture the super-convergent behavior. Both Mesh I and Mesh II have similar element size, element shape and density and differ mainly in the $\pi / 4$ rotation. The error equidistributing meshes (Mesh I) are displayed in Figures 6, 8,10 and 12. The super-convergent meshes (Mesh II) are displayed in Figures 7, 9, 11 and 13. The error profiles in 2, 3, 4 and 5 clearly show significant improvement in accuracy of Mesh II over Mesh I. The almost level error profile for Mesh I indicates an equidistribution of interpolation error evenly over all elements as predicted by our simple error model.

Note that Example 1 produces a simple radially symmetric mesh with a regular angular partition. Even in this simple case, a $\pi / 4$ rotation yields substantial improvement in approximation accuracy.

Results on Table 1 and Table 3 show the expected $O\left(h^{2}\right)$ convergence rate for Mesh I. A four-fold increase of elements leads to a four-fold decrease in error. Results for Mesh II demonstrate a higher than $O\left(h^{2}\right)$ convergence. A four-fold increase of elements leads to an eight fold decrease in error. This suggests $O\left(h^{3}\right)$ convergence behavior for Mesh II.

In summary, we have derived a simple error model for bilinear approximation over a parallelogram. We used this model to motivate the generation of super-convergent meshes using an anisotropic coordinate transformation of a regular mesh of squares. The numerical experiments clearly demonstrate the effectiveness of the super-convergent mesh for certain non-convex data functions. The insight gained here might have application to mesh design near known point or corner singularities. 
Table 3: Convergence test on Example 3.

\begin{tabular}{|l||c|c|c|c|r|}
\hline \hline & $\begin{array}{c}\text { Minimum } \\
\text { error }\end{array}$ & $\begin{array}{c}\text { Median } \\
\text { error }\end{array}$ & $\begin{array}{c}90 \\
\text { percentile }\end{array}$ & $\begin{array}{c}\text { Maximum } \\
\text { error }\end{array}$ & $\begin{array}{c}\text { Number of } \\
\text { elements }\end{array}$ \\
\hline \hline Mesh I & $1.51 \mathrm{e}+00$ & $1.51 \mathrm{e}+00$ & $1.52 \mathrm{e}+00$ & $1.56 \mathrm{e}+00$ & 255 \\
Mesh I & $4.54 \mathrm{e}-01$ & $4.54 \mathrm{e}-01$ & $4.54 \mathrm{e}-01$ & $4.60 \mathrm{e}-01$ & 916 \\
Mesh I & $1.13 \mathrm{e}-01$ & $1.13 \mathrm{e}-01$ & $1.14 \mathrm{e}-01$ & $1.15 \mathrm{e}-01$ & 3837 \\
Mesh I & $2.84 \mathrm{e}-02$ & $2.84 \mathrm{e}-02$ & $2.84 \mathrm{e}-02$ & $2.85 \mathrm{e}-02$ & 15685 \\
\hline Mesh II & $2.36 \mathrm{e}-02$ & $4.06 \mathrm{e}-02$ & $9.66 \mathrm{e}-02$ & $5.09 \mathrm{e}-01$ & 259 \\
Mesh II & $3.69 \mathrm{e}-03$ & $6.69 \mathrm{e}-03$ & $1.63 \mathrm{e}-02$ & $9.64 \mathrm{e}-02$ & 918 \\
Mesh II & $4.52 \mathrm{e}-04$ & $8.29 \mathrm{e}-04$ & $2.04 \mathrm{e}-03$ & $1.44 \mathrm{e}-02$ & 3834 \\
Mesh II & $5.53 \mathrm{e}-05$ & $1.03 \mathrm{e}-04$ & $2.54 \mathrm{e}-04$ & $1.92 \mathrm{e}-03$ & 15682 \\
\hline
\end{tabular}

Table 4: Summary of results for Example 4.

\begin{tabular}{|l||c|c|c|c|c|}
\hline \hline & $\begin{array}{c}\text { Minimum } \\
\text { error }\end{array}$ & $\begin{array}{c}\text { Median } \\
\text { error }\end{array}$ & $\begin{array}{c}90 \\
\text { percentile }\end{array}$ & $\begin{array}{c}\text { Maximum } \\
\text { error }\end{array}$ & $\begin{array}{c}\text { Number of } \\
\text { elements }\end{array}$ \\
\hline \hline Mesh I & $4.21 \mathrm{e}-4$ & $4.21 \mathrm{e}-4$ & $4.22 \mathrm{e}-4$ & $4.26 \mathrm{e}-4$ & 576 \\
Mesh II & $5.90 \mathrm{e}-6$ & $9.90 \mathrm{e}-6$ & $1.90 \mathrm{e}-5$ & $3.97 \mathrm{e}-5$ & 575 \\
\hline
\end{tabular}

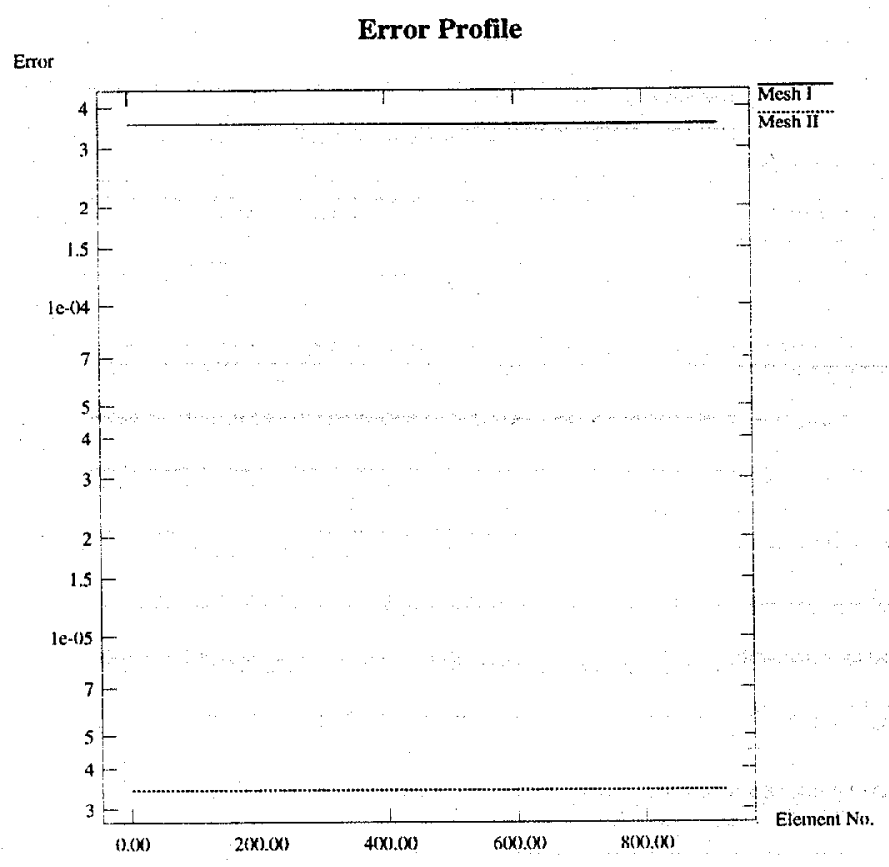

Figure 2: Error profiles for Example 1. 


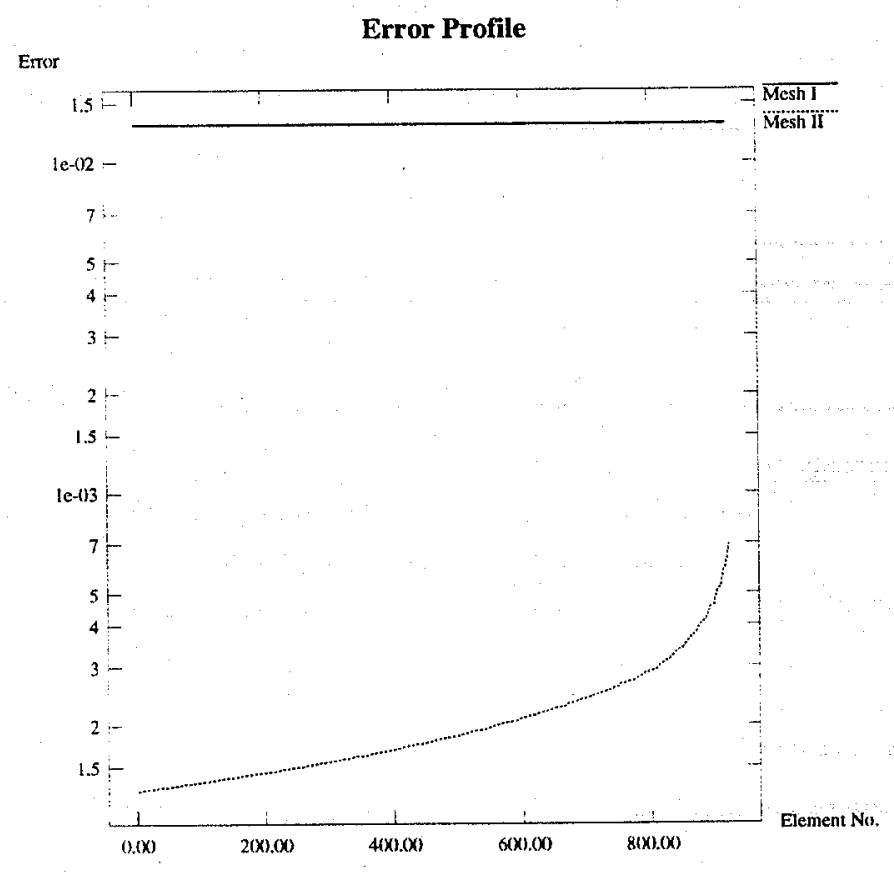

Figure 3: Error profiles for Example 2.

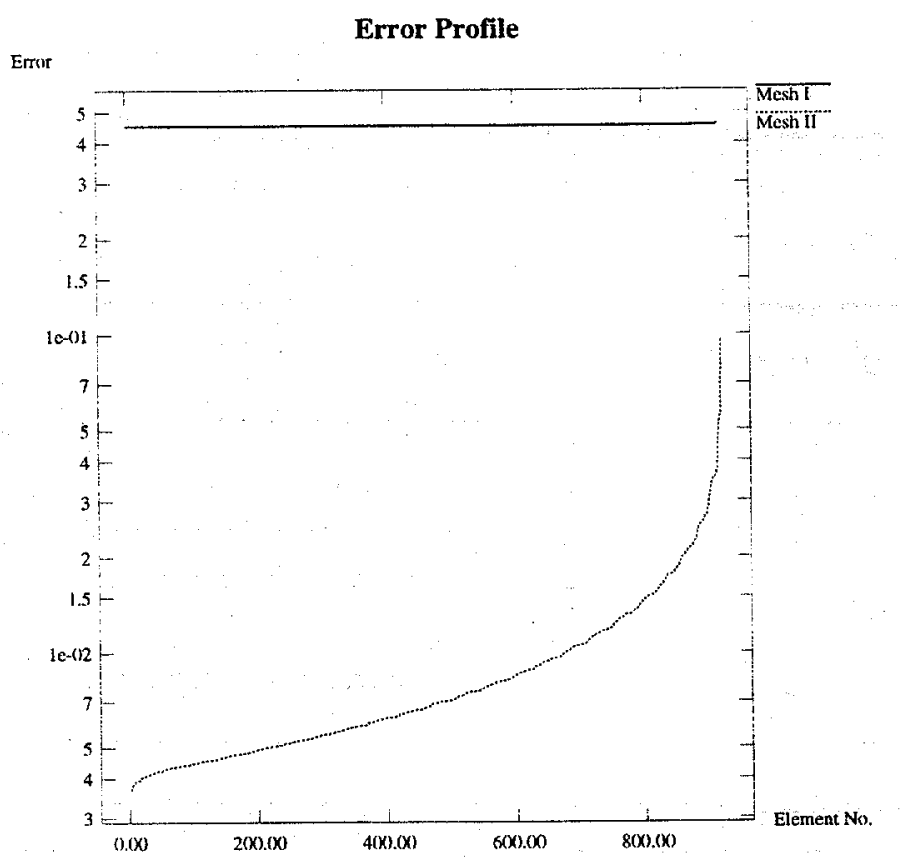

Figure 4: Error profiles for Example 3. 


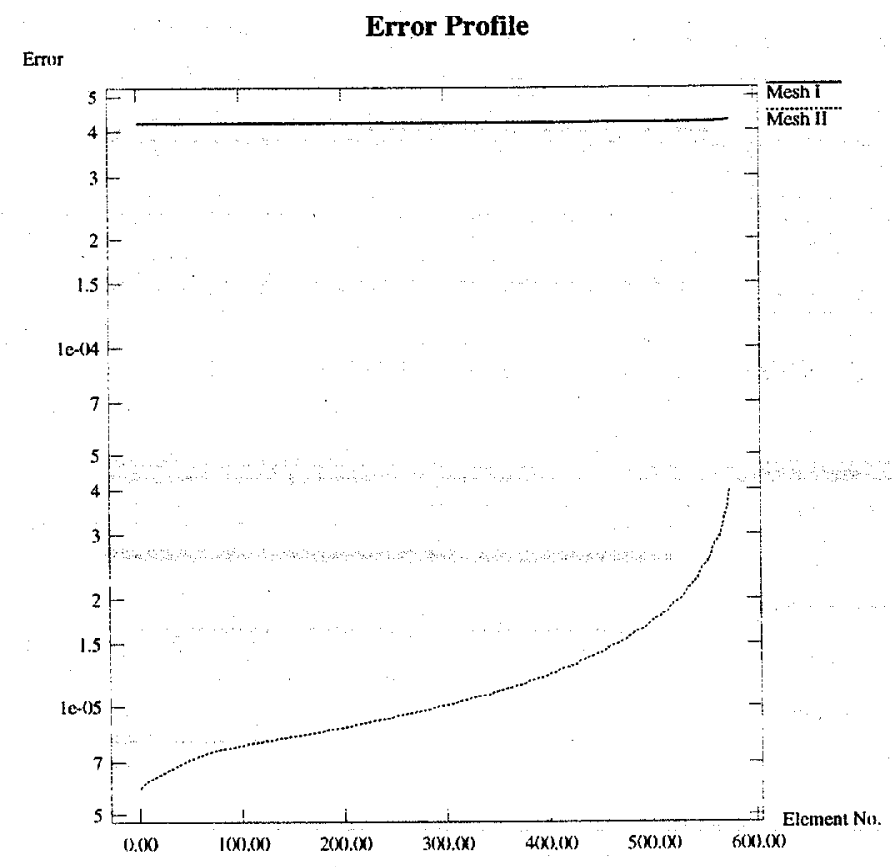

Figure 5: Error profiles for Example 4.

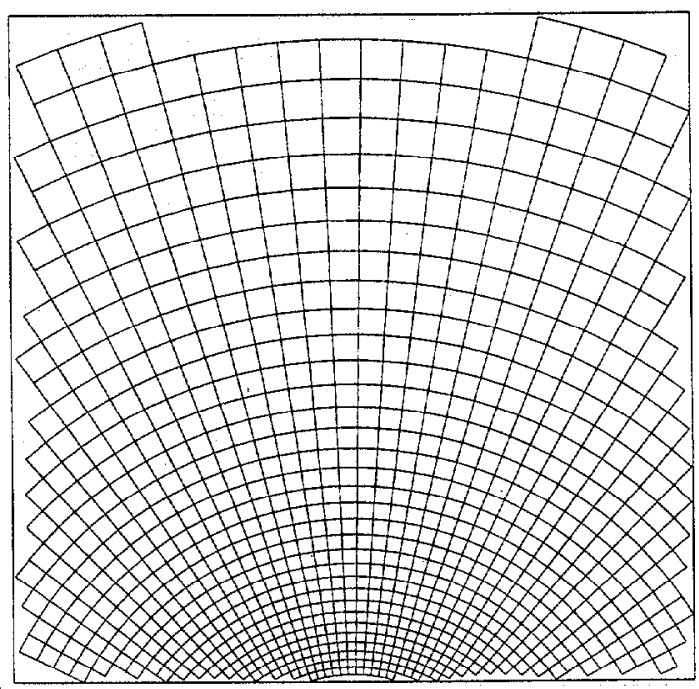

Figure 6: Mesh I for Example 1. 


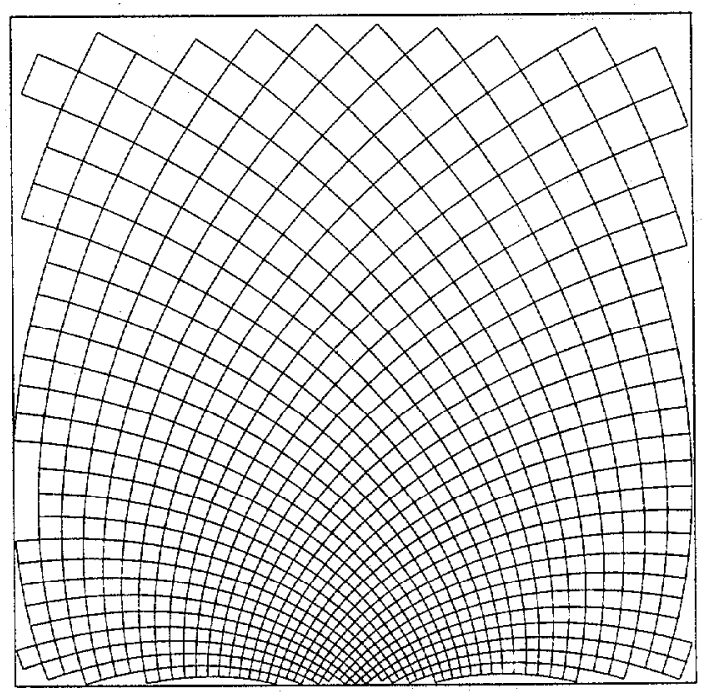

Figure 7: Mesh II for Example 1.

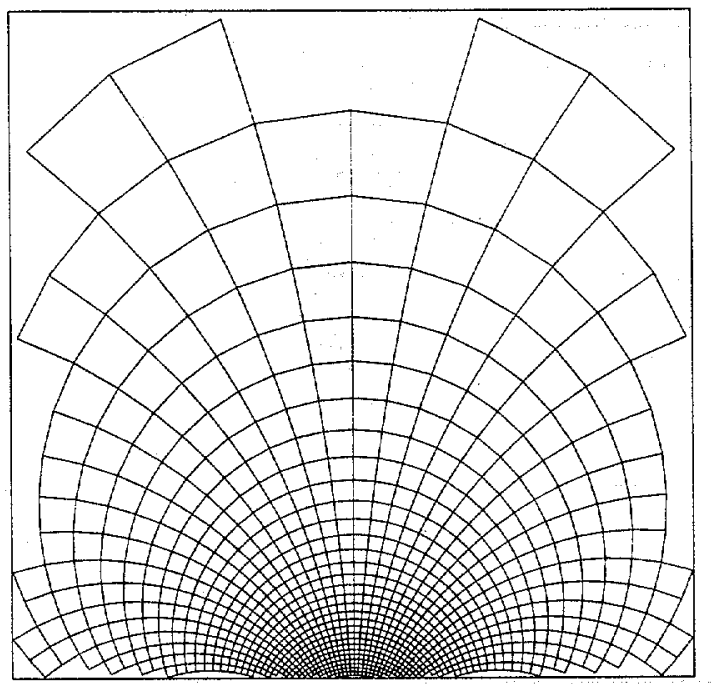

Figure 8: Mesh I for Example 2. 


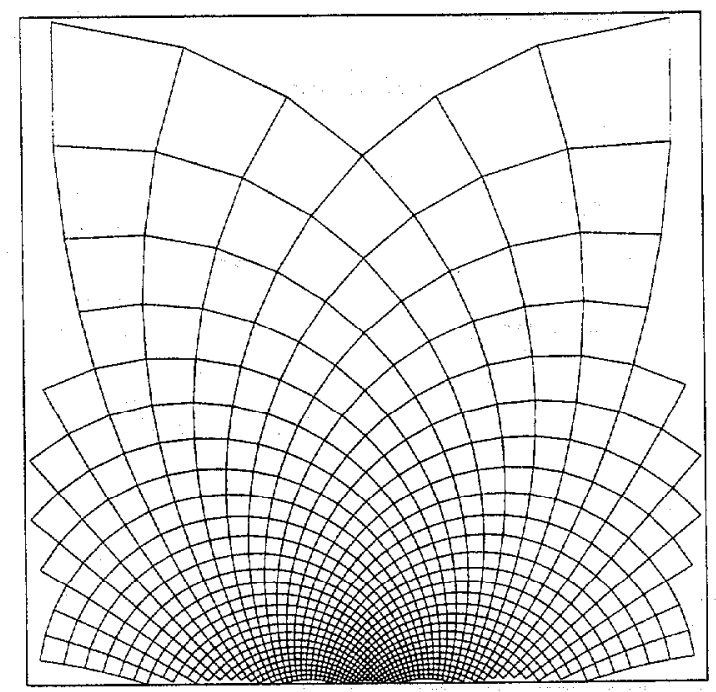

Figure 9: Mesh II for Example 2.

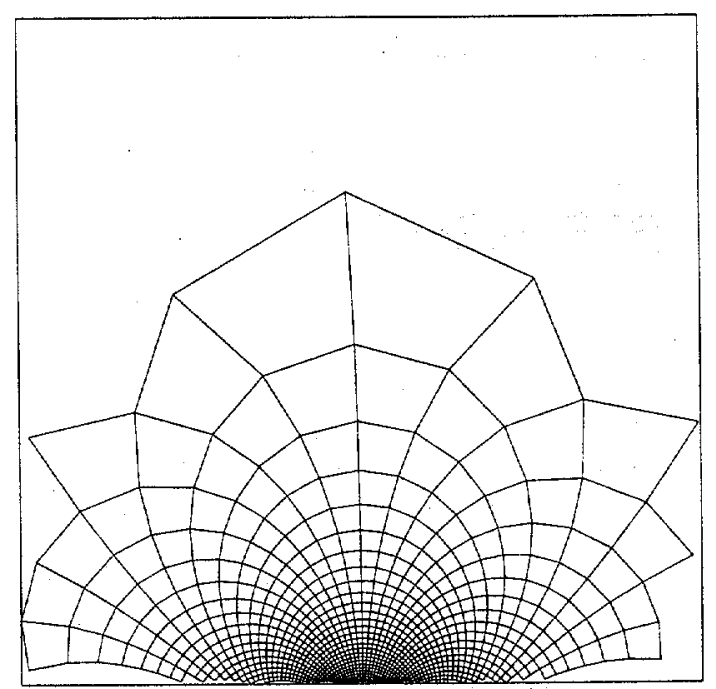

Figure 10: Mesh I for Example 3. 


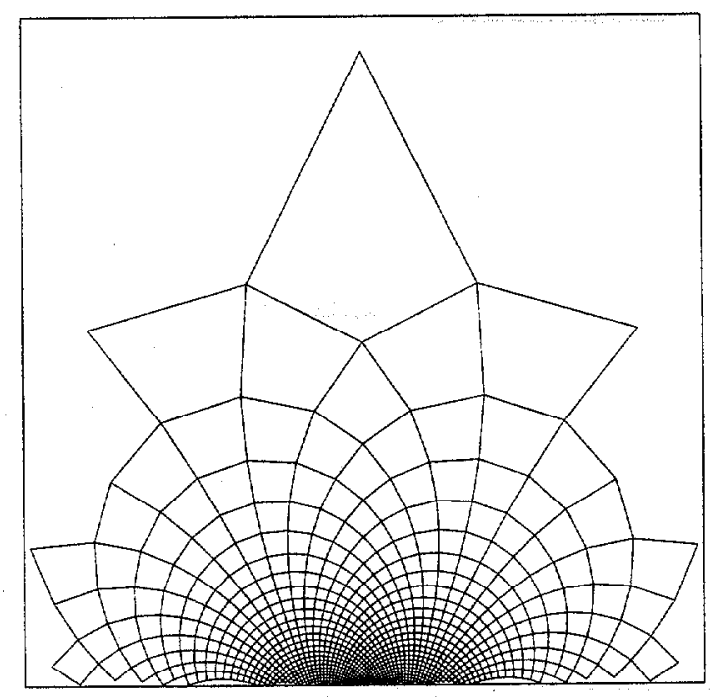

Figure 11: Mesh II for Example 3.

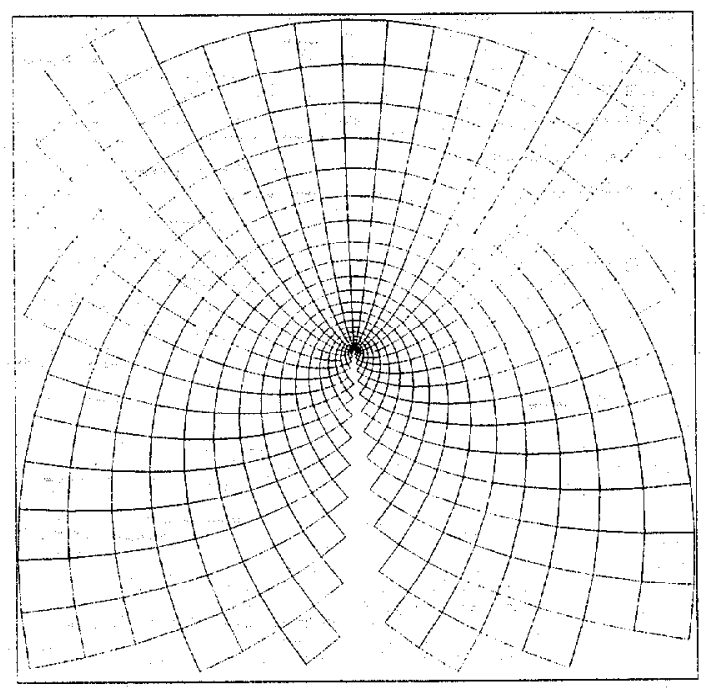

Figure 12: Mesh I for Example 4. 


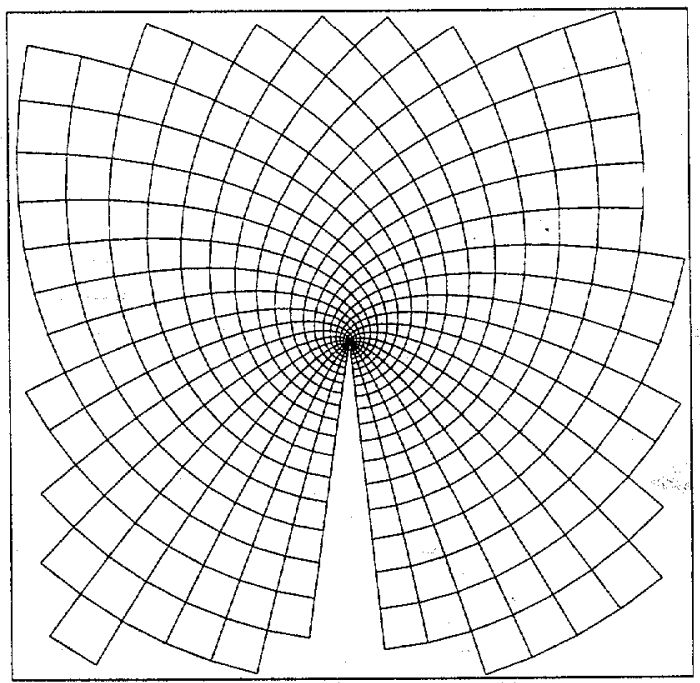

Figure 13: Mesh II for Example 4. 


\section{Appendix A}

In this section, we show the error function for quadratic interpolation over a parallelogram is given by (6) using only simple algebraic expansion. Let the data function be

$$
f(x, y)=\frac{1}{2}[x, y] H[x, y]^{t}+\left[g_{1}, g_{2}\right][x, y]^{t}+c
$$

and the affine isoparametric transformation be

$$
\left[\begin{array}{l}
x(p, q) \\
y(p, q)
\end{array}\right]=T\left[\begin{array}{l}
p \\
q
\end{array}\right]+\left[\begin{array}{l}
x_{1} \\
y_{1}
\end{array}\right], T=\left[\begin{array}{ll}
u_{x} & v_{x} \\
u_{y} & v_{y}
\end{array}\right]=\left[\begin{array}{ll}
x_{2}-x_{1} & x_{4}-x_{1} \\
y_{2}-y_{1} & y_{4}-y_{1}
\end{array}\right]
$$

Then the interpolation error can be shown to be

$$
\begin{aligned}
E_{Q}(p, q) & =p_{b}(x(p, q), y(p, q))-f(x(p, q), y(p, q)) \\
& =\mathcal{E}_{Q}-\frac{1}{2}\left(\mu_{1}\left(p-p_{c}\right)^{2}+\mu_{2}\left(q-q_{c}\right)^{2}\right)
\end{aligned}
$$

with centroid at $\left[p_{c}, q_{c}\right]=\left[\frac{1}{2}, \frac{1}{2}\right]$

$$
\begin{aligned}
\mathcal{E}_{Q} & =E_{Q}\left(p_{c}, q_{c}\right)=\frac{1}{8}\left(\mu_{1}+\mu_{2}\right) \\
\mu_{1} & =\left[u_{x}, u_{y}\right] H\left[u_{x}, u_{y}\right]^{t}, \quad \mu_{2}=\left[v_{x}, v_{y}\right] H\left[v_{x}, v_{y}\right]^{t}
\end{aligned}
$$

Let the data function over $(p, q)$-space be written as

$$
\begin{aligned}
& \begin{aligned}
\tilde{f}(p, q) & =f(x(p, q), y(p, q)) \\
& =\frac{1}{2}[p, q] \tilde{H}[p, q]^{t}+\left[\tilde{g}_{1}, \tilde{g}_{2}\right][p, q]^{t}+\tilde{c}
\end{aligned} \\
& \text { where } \tilde{H}=T^{t} I I T-\left[\begin{array}{ll}
\tilde{h}_{11} & \tilde{h}_{12} \\
\tilde{h}_{12} & \tilde{h}_{22}
\end{array}\right] \text { and } \\
& {\left[\tilde{g}_{1}, \tilde{g}_{2}\right]=\left(\left[g_{1}, g_{2}\right]+\left[x_{1}, y_{1}\right] H\right) T,} \\
& \tilde{c}=c+\left[g_{1}, g_{2}\right]\left[x_{1}, y_{1}\right]^{t}+\frac{1}{2}\left[x_{1}, y_{1}\right] H\left[x_{1}, y_{1}\right]^{t} .
\end{aligned}
$$

The function values at the four interpolating corners are

$$
\begin{aligned}
& f_{1}=\tilde{f}(0,0)=\tilde{c}, \quad f_{3}=\tilde{f}(1,1)=\frac{1}{2}\left(\tilde{h}_{11}+\tilde{h}_{22}+2 \tilde{h}_{12}\right)+\tilde{g}_{1}+\tilde{g}_{2}+\tilde{c}, \\
& f_{2}=\tilde{f}(1,0)=\frac{1}{2} \tilde{h}_{11}+\tilde{g}_{1}+\tilde{c}, \quad f_{4}=\tilde{f}(0,1)=\frac{1}{2} \tilde{h}_{22}+\tilde{g}_{2}+\tilde{c} .
\end{aligned}
$$

By (5) and (16) (note the vanishing of linear and constant terms),

$$
\begin{aligned}
E_{Q}(p, q)= & \left(\sum_{i=1}^{i=4} f_{i} \phi_{i}(p, q)\right)-\tilde{f}(p, q) \\
= & \frac{1}{2}\left(p(1-q) \tilde{h}_{11}+p q\left(\tilde{h}_{11}+\tilde{h}_{22}+2 \tilde{h}_{12}\right)\right. \\
& \left.\quad+(1-p) q \tilde{h}_{22}-\left(p^{2} \tilde{h}_{11}+q^{2} \tilde{h}_{22}+2 p q \tilde{h}_{12}\right)\right) \\
= & \frac{1}{2}\left(p \tilde{h}_{11}+q \tilde{h}_{22}+2 p q \tilde{h}_{12}-p^{2} \tilde{h}_{11}-q^{2} \tilde{h}_{22}-2 p q \tilde{h}_{12}\right) \\
= & \frac{1}{2}\left(p(1-p) \tilde{h}_{11}+q(1-q) \tilde{h}_{22}\right) \\
= & \frac{1}{8}\left(\tilde{h}_{11}+\tilde{h}_{22}\right)-\frac{1}{2}\left(\tilde{h}_{11}\left(p-\frac{1}{2}\right)^{2}+\tilde{h}_{22}\left(q-\frac{1}{2}\right)^{2}\right) .
\end{aligned}
$$

From (15) and (17), we have $\tilde{h}_{11}=\mu_{1}$ and $\tilde{h}_{22}=\mu_{2}$; hence the error function has the form given in (16). 


\section{References}

[1] Houman Borouchaki, Paul Louis George, Frederic Hecht, Patrick Laug, and Eric Saltel. Delaunay mesh generation governed by metric specifications. Part I. Algorithms. Finite Elements in Analysis and Design, 25:61-83, 1997.

[2] E. F. D'Azevedo. Optimal triangular mesh generation by coordinate transformation. SIAM J. Sci. Statist. Comput., 12(4):755-786, 1991.

[3] E. F. D'Azevedo and R. B. Simpson. On optimal interpolation incidences. SIAM J. Sci. Statist. Comput., 10:1063$1075,1989$.

[4] E. F. D'Azevedo and R. B. Simpson. On optimal triangular meshes for minimizing the gradient error. Numer. Math., 59:321-348, 1991.

[5] A. R. Mitchell and R. Wait. The Finite Element Method in Partial Differential Equations. Wiley-Interscience Publication, 1977.

[6] E. Nadler. Piecewise linear best $l_{2}$ approximation on triangulations. In C. K. Chui, L. L. Schumaker, and J. D. Ward, editors, Approximation Theory V, pages 499-502, Boston, 1986. Academic Press.

[7] J. Peraire, M. Vahdati, KMorgan, and O. C. Zienkiewics. Adaptive remeshing for compressible flow computations. J. Comput. Phys., 72:449-466, 1987.

[8] Kenji Shimada. Anisotropic triangular meshing of parametric surfaces via close packing of ellipsoidal bubbles. In Proceedings 6th International Meshing Roundtable 1997, October 1997, Park City, Utah, 1997. Also available as Sandia Report SAND 97-2399 UC-405.

[9] R. B. Simpson. Anisotropic mesh transformations and optimal error control. Applied Numerical Mathematics, 1992. Speical issue as the proceedings of the US Army sponsored Workshop for Adaptive Methods for Partial Differential Equations, Rensselaer Polytechnical Institute (accepted).

[10] I. S. Sokolnikoff. Tensor Analysis, Theory and Applications to Geometry and Mechanics of Continua. John Wiley, New York, second edition, 1964. 